\section{Erhöhte Rate von Leberabszessen im Endo Trial}

Wie aber sieht es mit der Sicherheit des Systems aus? Komplikationen, die zum Entfernen des EndoBarriers ${ }^{\circledast}$ geführt haben, seien u. a. gastrointestinale Blutungen (1,5\%), Unverträglichkeiten $(1,6 \%)$, Migration $(1,6 \%)$ und Leberabszesse $(0,9 \%)$, berichtete PD Dr. Erhard Siegel, Chefarzt am St. Josefskrankenhaus in Heidelberg. Die unerwartet hohe Rate an Leberabszessen im USamerikanischen „ENDO Trial“ (bei 3,5\% der 201 Typ-2-Diabetiker) habe dazu geführt, dass der Hersteller die Studie im Juli 2015 vorzeitig abgebrochen hat, so der Gastroenterologe. Leberabszesse seien hier jedoch deutlich häufiger aufgetreten, als es sonst in der Klinik beobachtet werde.

Weitere Datenanalysen würden derzeit noch laufen und der FDA zeitnah übermittelt, so Siegel. Dass es bisher noch keine großen randomisierten Studien zur Sicherheit und Effektivität des Systems gibt, gibt auch Laubner zu denken. Langzeitdaten zum Gewichts- und $\mathrm{HbA}_{1 \mathrm{c}}$-Verlauf nach Explantation des Systems oder zur Bioverfügbarkeit von Medikamenten liegen noch nicht vor.

Laubner hofft deshalb, dass sich die Datenlage bald verbessern wird und verweist in diesem Zusammenhang auf die noch für dieses Jahr erwartete erste Auswertung des nationalen EndoBarrier-Registers unter Leitung von Prof. Jochen Seufert und Prof. Jens Aberle. Dann, so hofft die Diabetologin, werde sich das Device in erfahrenen Zentren vielleicht als wertvolle Therapieergänzung für ausgewählte adipöse Typ-2-Diabetiker etablieren können.

Veronika Schlimpert

Quelle: Symposium „Interventionelle Therapie des Typ-2-Diabetes", 6. Mai

2016, DDG-Jahrestagung, Diabeteskongress in Berlin

\title{
Das Hirn arbeitet nach Magen-Bypass anders
}

\author{
Eine Bypass-Operation wirkt sich neben der Gewichtsregulation offenbar auch auf \\ die neuronale Verarbeitung von Nahrungsreizen aus, wie eine Tübinger Arbeitsgruppe \\ nun beobachtet hat.
}

Nach einer bariatrischen Bypass-Operation verändert sich bei Typ-2-Diabetikern offenbar die Aktivität in für die Nahrungsverarbeitung wichtigen Gehirnarealen. Diese Beobachtung machten Tübinger Wissenschaftler um Dr. Sabine Frank, nachdem sie 24 adipöse Typ-2-Diabetiker, davon 12 operierte und 12 Kontrollpatienten, während einer funktionellen Magnetresonanztomografie Bilder verschiedener Nahrungsmittel gezeigt haben. Die Probanden sollten ihr Verlangen („Wanting“) und generelle Vorliebe („Likeing“) für diese Speisen bewerten. Zuvor waren sie auf Alter, BMI und präoperatives $\mathrm{HbA}_{1 \mathrm{c}}$ gematcht worden. Wie in früheren Studien schon beobachtet worden sei, habe sich sowohl das Verlangen als auch die generelle Vorliebe für hochkalorische Speisen wie Donuts oder Burger in der bariatrischen Gruppe signifikant verringert, berichtete Frank beim Diabeteskongress.

\section{Veränderungen in belohnungssensitiven Gehirnarealen}

Diese veränderte Nahrungspräferenz geht offenbar mit einer veränderten neuronalen Aktivität einher. So fand sich im MRT bei den operierten Patienten im Vergleich zu Kontrollgruppe eine verstärkte Aktivität im gustatorischen Kortex, der für die Verarbeitung nahrungsassoziierter Reize zuständig ist, im Frontalkortex - wichtig für die kognitive Kontrolle - sowie im Hippocampus, einem insulinsensitiven Areal. Die Aktivität in belohnungssensitiven Gehirnarealen wie dem Inferioren Frontalkortex und Globus pallidus war dagegen in der Bypass-Gruppe reduziert und somit die Belohnungsaktivität bei Nahrungsstimulation bei diesen Patienten offensichtlich gehemmt. Die nach der Bypass-OP beobachtete $\mathrm{HbA}_{1 \mathrm{c}}$-Senkung korreliere zudem mit der Aktivität des orbitofrontalen Kortex, in dem vor allem
Der Denk- und der Verdauungsapparat sind eng verknüpft. Hier gibt's noch viel Forschungsbedarf.

die Evaluation und Integration von Informationen stattfinde, berichtete Frank.

„Also je stärker

die Verbesserung

der glykämischen

Kontrolle, desto stär-

ker die integrative und evaluative Verarbeitung von Nahrungsreizen." Somit könnten auch neuronale Aktivitätsveränderungen im Gehirn eine wichtige Rolle für die langanhaltende Dia-

betesremission spielen, schlussfolgerte Frank. In einer Langzeitstudie sollte geprüft werden, welche Faktoren im Zusammenhang der Bypass-OP - ob die langfristige Gewichtsreduktion oder andere Mechanismen in der Initialphase der Stoffwechselverbesserung durch eine erhöhte Inkretinsekretion - zu diesen neuronalen Veränderungen geführt haben.

Veronika Schlimpert

Quelle: Symposium „Interventionelle Therapie des Typ-2-Diabetes",

06.05.2016, DDG-Jahrestagung in Berlin 